\title{
INCIDÉNCIA E CARACTERISTICAS DA SEPSE EM UMA UNIDADE DE TERAPIA INTENSIVA DE UM HOSPITAL MISTO DO PARANÁ
}

\author{
Sepsis incidence and characteristics in an Intensive \\ Care Unit of a mixed hospital in the state of Parana
}

Estefani Teresinha Seibt ${ }^{1}$, Joice Cristina Kuchler ${ }^{2}$, Franciele do Nascimento Santos Zonta ${ }^{3}$

1. Enfermeira graduada pela Universidade Paranaense - Unidade Universitária de Francisco Beltrão PR. ORCID: https://orcid.org/0000-0002-5078-2915.

2. Enfermeira, discente do Programa de Mestrado em Ciências da Saúde - Universidade Estadual do Oeste do Paraná. ORCID: https://orcid.org/0000-0003-1515-3563.

3. Mestre em Ciências da Saúde pela Escola de Enfermagem da USP, Docente da Universidade Paranaense, Unidade de Francisco Beltrão. ORCID: https://orcid.org/0000-0002-4236-4027.

CONTATO: Joice Cristina Kuchler | Endereço: Cabeceira do Lontra | Nova Esperança do Sudoeste - PR | Telefone: (46) 984214762 | E-mail: joicekuchler97@gmail.com

COMO CITAR: Seibt ET, Kuchler JC, Zonta FNS. Incidência e características da sepse em uma unidade de terapia intensiva de um hospital misto do Paraná. R. Saúde Públ. Paraná. 2019 Dez;2(2):97-106.

\footnotetext{
(c) (1)

COPYRIGHT Esta obra é disponibilizada nos termos da Licença Creative Commons - 4. 0

Internacional. É permitida a reprodução parcial ou total desta obra, desde que citada a fonte.
}

RESUMO A sepse e o choque séptico são as maiores causas de óbito em pacientes internados em Unidades de Terapia Intensiva, devido à dificuldade na sua identificação e ao tratamento minucioso exigido. Objetivando identificar o índice e as características da sepse em uma UTI adulto de um hospital do Paraná, realizou-se uma pesquisa de campo retrospectiva, documental e quantitativa. A amostra foi constituída de 432 prontuários. Dados foram coletados por meio de um checklist próprio. Houve prevalência do sexo masculino e faixa etária acima dos cinquenta anos. A etiologia de admissão prevalente foi insuficiência respiratória e broncopneumonia. Quanto ao foco infeccioso, 
prevaleceu o pulmonar. Constatou-se índice elevado dos casos de sepse, juntamente de uma taxa de mortalidade ultrapassando a média geral do país. Sugere-se uma monitorização e notificação mais eficaz dos casos, combinada com a aplicação de um protocolo unificado, visando à redução da taxa de letalidade por esta síndrome.

PALAVRAS-CHAVE: Sepse. Unidades de Terapia Intensiva. Infecção Hospitalar. Epidemiologia. Mortalidade.

ABSTRACT Sepsis and septic shock are the major causes of death in patients admitted to intensive care units, due to the difficulty in identifying them and the thorough treatment required. In order to identify the index and characteristics of sepsis in an adult ICU of a hospital in the state of Paraná, a retrospective, documentary and quantitative field research was carried out. The sample consisted of 432 medical records. Data were collected through their own checklist. There was a male prevalence and age group above fifty years. The prevalent etiology of admission was respiratory failure and bronchopneumonia. Regarding the infectious focus, the pulmonary prevailed. A high rate of sepsis cases was found, together with a mortality rate exceeding the general average of the country. More effective monitoring and reporting of cases is suggested, combined with the application of a unified protocol to reduce the case fatality rate for this syndrome.

KEYWORDS: Sepsis. Intensive Care Units. Cross Infection. Epidemiology. Mortality.

\section{INTRODUÇÃO}

$\mathbf{A}$

s Unidades de Terapia Intensiva (UTI) são ambientes complexos e dinâmicos, que atendem pacientes em estado grave, representado por doenças adquiridas, traumas ou pós-operatórios, apresentando elevado risco de óbito. Para o manejo dos pacientes, a UTI disponibiliza equipe multiprofissional, materiais e recursos adequados, que permitem a observação integral e contínua dos pacientes. Dessa forma, é possível garantir o atendimento de forma ágil e eficaz em prol do restabelecimento e promoção da saúde dos mesmos 1 .
Por outro lado, é um local com elevado índice de óbitos entre todos os setores hospitalares. Devido à complexidade dos casos, ao longo do tempo foram implantados novos procedimentos terapêuticos, cada vez mais invasivos, o que favorece o desenvolvimento de infecções graves, bem como o uso indiscriminado de antimicrobianos, o que vem tornando os micro-organismos multirresistentes ${ }^{2}$.

Atualmente, a sepse é a maior causa de óbitos em ambientes hospitalares, especialmente na UTI, devido à dificuldade na sua identificação 
e ao tratamento minucioso que exige agilidade e precisão no momento do diagnóstico. Além disso, o custo com exames, materiais, procedimentos e medicamentos também é substancial, tornando a sepse um problema mundial de saúde, com repercussão no cenário econômico ${ }^{3}$.

Após o consenso entre a Society of Critical Care Medicine (SCCM) e a European Society of Critical Care Medicine (ESICM), a sepse passou a ser definida como uma disfunção orgânica agressivamente letal, decorrente de uma resposta desregulada do hospedeiro frente a uma infecção ${ }^{4}$. Tal condição é conhecida mundialmente por sua gravidade e letalidade por evoluir, na maioria dos casos, com disfunção de múltiplos órgãos, especialmente rins e pulmão, comprometendo gravemente o prognóstico do paciente?

No Brasil, a sepse ultrapassa 400.000 casos por ano, acometendo hospitais dos mais diferentes portes, públicos ou privados. Em um hospital do interior do Paraná foi realizado um estudo epidemiológico, caracterizando um quinquênio da sepse, e observou-se uma incidência de $71,4 \%$ entre os pacientes internados na UTI, com taxa de mortalidade de $63,4 \%{ }^{4}$.

Vale ressaltar que, em todo o Brasil, o índice de óbitos causados pela síndrome é elevado. A incidência é de aproximadamente 200 mil casos por ano, com taxas de mortalidade de 35 a $65 \%{ }^{5}$.

As características clínicas da sepse e os fatores associados ao estado de saúde do paciente são determinantes para o diagnóstico precoce e intervenção adequada. Por isso, a equipe multiprofissional deve compreender os riscos e consequências da sepse. A partir do olhar clínico destes profissionais, embasado nas evidências epidemiológicas, é possível em tempo hábil tomar iniciativas terapêuticas capazes de minimizar as complicações, impedindo que o quadro se agrave e evolua com prognóstico ruim ${ }^{6}$.

Apesar da disseminação de algumas informações, ainda se fazem necessários estudos que optem por discutir sobre a gravidade, manejo e incidência da sepse. O conhecimento sobre a síndrome, além de mostrar a preocupação gerada ao âmbito da saúde, esclarece a elevada taxa de óbitos em UTIs ${ }^{3}$. Dessa forma, o objetivo deste estudo foi identificar o índice e as características da sepse bem como descrever suas complicações, apontando o índice de sobrevida dos pacientes acometidos por tal patologia que estavam internados em uma UTI adulto de um hospital misto do Paraná.

\section{METODOLOGIA}

Trata-se de uma pesquisa de campo exploratória, documental e retrospectiva, com abordagem quantitativa, que compreendeu um levantamento dos pacientes que estiveram internados em uma UTI adulto, no período de janeiro de 2015 a janeiro de 2018. O estudo foi realizado em um hospital geral de médio porte, tendo como gestor o poder público e privado.

A seleção dos prontuários dos pacientes com sepse na UTI obedeceu aos critérios de inclusão: prontuários de pacientes maiores de 18 anos de ambos os sexos, com ficha de Internação hospitalar preenchida, internados no período de janeiro de 2015 a janeiro de 2018, com diagnóstico de sepse. E como critérios de exclusão: prontuários indisponíveis no período de realização da pesquisa ou incompletos, bem como pacientes sem diagnóstico de sepse.

Para a obtenção dos dados foi utilizado um checklist, confeccionado a partir de variáveis descritas na literatura, a saber: sexo, idade, dias hospitalizado, tipo de paciente (clínico, cirúrgico ou trauma), etiologia de admissão na UTI, presença de hipertermia, hipotermia, taquicardia, taquipneia, leucocitose, leucopenia, administração de antimicrobiano em possível foco infeccioso, sinais de disfunção orgânica como Pressão Arterial Sistólica (PAS) $<90 \mathrm{mmHg}$ ou Pressão Arterial Média (PAM) $<65$, queda de Pressão Arterial (PA) $>40 \mathrm{mmHg}$ Creatinina $>2.0 \mathrm{mg} / \mathrm{dl}, \quad$ Plaquetas $<100.000$ 
proteína C reativa, recente necessidade de uso de gás oxigênio $\left(\mathrm{O}_{2}\right)$ para manter a Saturação Percutânea de Oxigênio $(\mathrm{SpO})$ >90, administração de vasopressores, critérios diagnósticos para SIRS, sepse, sepse grave ou choque séptico, origem da sepse, e o desfecho final (alta ou óbito).

Para a obtenção dos prontuários foi realizada uma pesquisa no programa SP Data ${ }^{\circledR}$. de uso interno do hospital, o qual contém dados pertinentes à internação dos pacientes conforme a especialidade do atendimento. A partir disso, foi possível delimitar somente os pacientes que estiveram internados na UTI, cujo diagnóstico estabeleceu critérios compatíveis com a sepse. Então foi realizada a busca em cada prontuário, seguindo rigidamente os critérios de inclusão e exclusão do estudo.

O processo de coleta e análise dos dados ocorreu nos meses de maio a julho de 2018, e subsequentemente foi realizada a análise descritiva, pelo software Statistical Package for Social Science (SPSS) versão $21.0 \AA$.

Para consolidação da pesquisa foram regidos e seguidos todos os preceitos éticos e legais conforme as Diretrizes da Resolução 466/12, do Conselho Nacional de Saúde?. Desta forma, obtevese a Declaração de Permissão de Utilização de Dados da referida instituição. Posteriormente, o projeto foi submetido ao Comitê de Ética em Pesquisa Envolvendo Seres Humanos da Universidade Paranaense e aprovado sob Protocolo n'2.565.444/2018.

\section{RESULTADOS}

Entre os anos de 2015 e 2018, 1.188 pacientes tinham registros de internação na UTI, e, destes, 432 (36,4\%) apresentaram sepse. Observou-se que 248 (57,5\%) pacientes eram do sexo masculino e $183(42,5 \%)$ do sexo feminino, com média de idade variando entre 51 e 60 anos (145; 33,6\%). A maior frequência de internamentos foi por condições cirúrgicas (47,3\%). Como etiologia de admissão, houve a prevalência dos casos de insuficiência respiratória aguda e broncopneumonia (129; 29,9\%). Quanto às doenças crônicas associadas, 289 (66,8\%) não possuíam nenhuma comorbidade associada, porém a Hipertensão Arterial Sistêmica (HAS) foi a doença crônica mais frequente (14,1\%) entre os pacientes, conforme a Figura 1.

Figura 1 Dados referentes ao perfil prevalente dos pacientes diagnosticados com sepse, internados em uma UTI adulto de um hospital misto do Paraná, entre janeiro de 2015 e janeiro de 2018

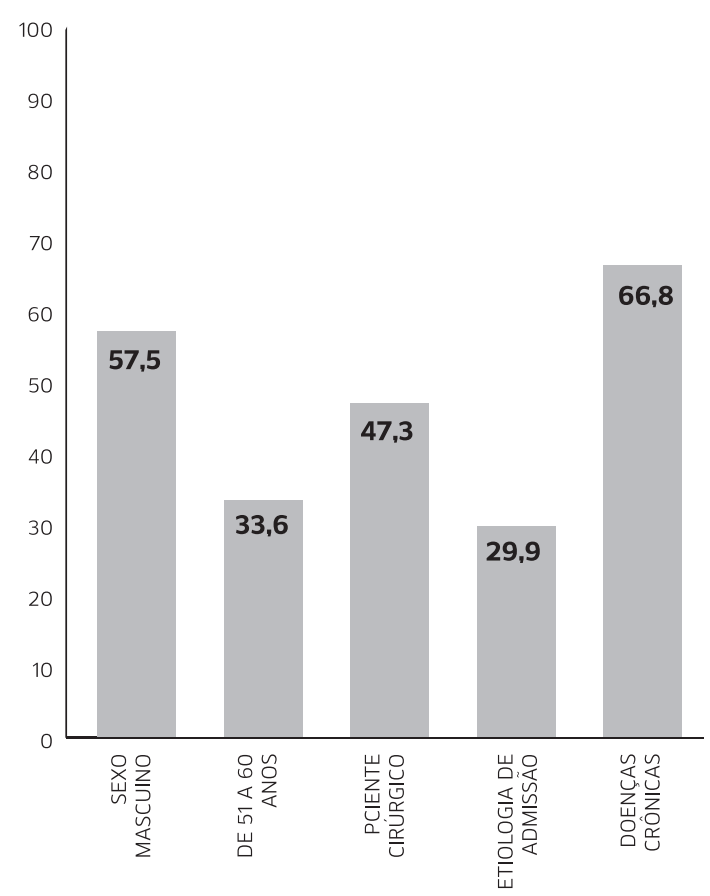

Fonte: Elaborado pelos autores (2018)

Os 432 pacientes que foram diagnosticados com sepse apresentaram sinais clínicos inerentes ao quadro (Gráfico 2), no qual se verificou que 66,6\% apresentaram hipertermia e 36,9\% hipotermia. A taquicardia ocorreu em 341 (79,1\%) pacientes e a taquipneia em 260 (60,3\%). 
Figura 2 Dados inerentes a manifestações clínicas do quadro séptico apresentados pelos pacientes envolvidos na pesquisa.

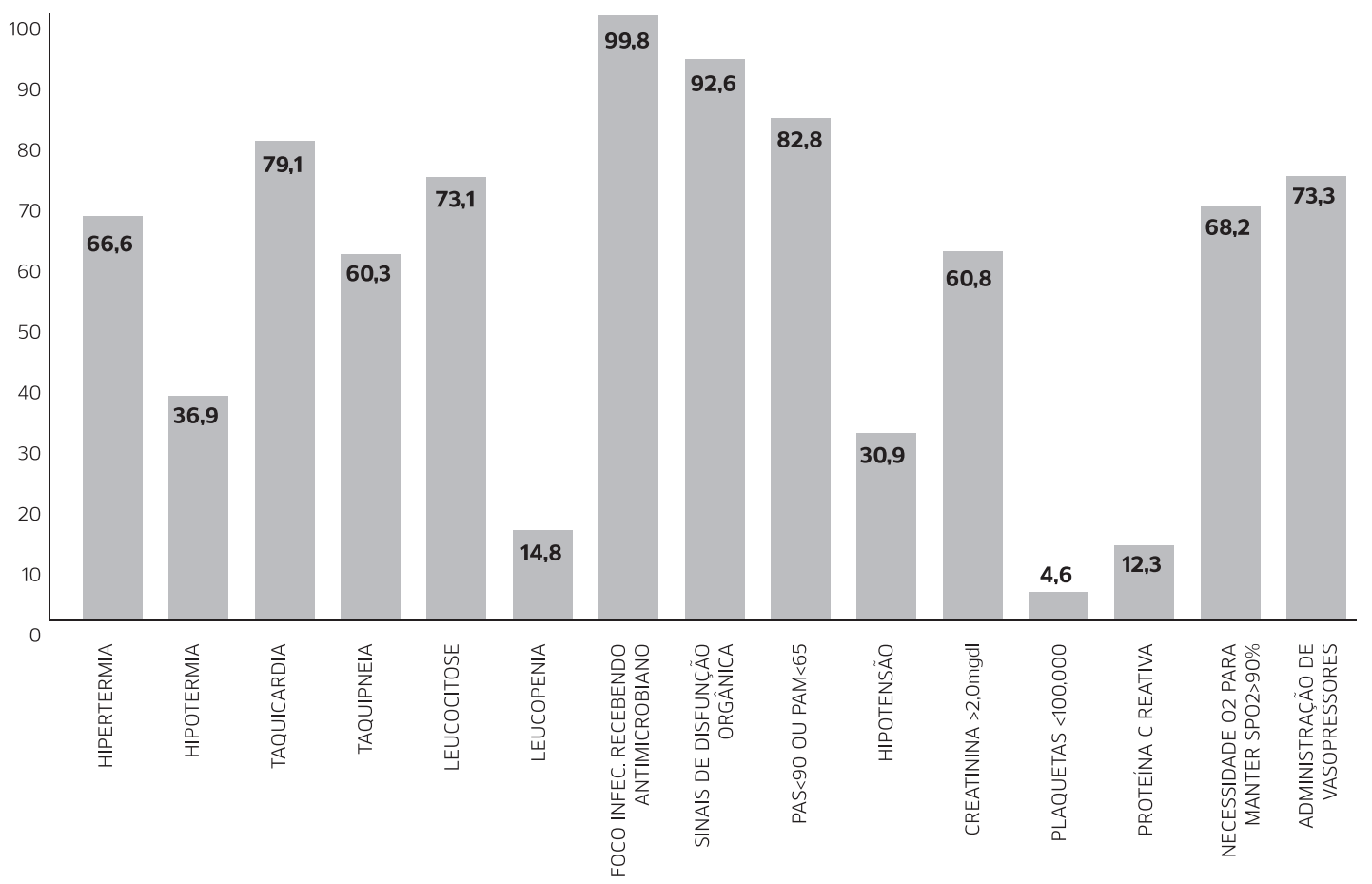

Fonte: Elaborado pelos autores (2018)

A contagem dos leucócitos, que é um fator para diagnóstico de infecção, mostrou que 315 (73,1\%) apresentaram leucocitose e 64 (14,8\%), leucopenia. Quanto à administração de antimicrobiano com foco infeccioso suspeito ou confirmado, 430 (99,8\%) já estavam recebendo e apenas um (0,2\%) não recebeu antibioticoterapia no momento do diagnóstico.

Os sinais clínicos de disfunção orgânica mostraram-se em 399 (92,6\%) pacientes, ou seja, um número elevado de complicações, e somente 32 (7,4\%) não os apresentaram. Ainda dentre estes pacientes, foi observado que durante a internação na UTI e após a instalação do quadro séptico, 357 mostraram PAS <90mmHg ou PAM <65 (82,8\%).

A hipotensão ocorreu em 357 (82,8\%) pacientes. A contagem de creatinina $>2.0 \mathrm{mgdl}$, ocorreu em 262 (60,8\%) do total de 432 internados. A contagem das plaquetas também é um fator contribuinte no diagnóstico, porém, apenas 20 $(4,6 \%)$ dos pacientes apresentaram plaquetas $<100.000$.

A contagem da Proteína C Reativa foi realizada em 53 (12,3\%) pacientes e todos apresentaram resultado reagente. Quanto à necessidade do suporte de $\mathrm{O}_{2}$ para manterem $\mathrm{SpO}_{2}>90 \%$, foi necessário em 294 (68,2\%) pacientes. Quando se instala a hipotensão severa faz-se necessária a administração de drogas vasoativas, desta forma, as medicações vasopressoras foram utilizadas em $316(73,3 \%)$ casos.

A partir desses sinais e sintomas clínicos, 
foram obtidos os critérios diagnósticos, em que 115 (26,6\%) indicaram sepse e 316 (73,3\%) choque séptico. A origem da sepse ocorre de duas formas, e neste estudo 259 (60,1\%) foram comunitárias e 172 (39,9\%) nosocomiais. O foco infeccioso pulmonar foi o prevalente entre os pacientes com sepse (342;
79,1\%), seguido do urinário e abdominal (78:18,0\% e $11 ; 2,54 \%$, respectivamente).

O desfecho dos quadros de sepse mostrou que 198 (45,9\%) pacientes receberam alta da UTI, enquanto 233 (54.1\%) foram a óbito, conforme mostra a figura 3.

Figura 3 Características e desfecho dos quadros de sepse apresentados pelos pacientes, entre janeiro de 2015 e janeiro de 2018

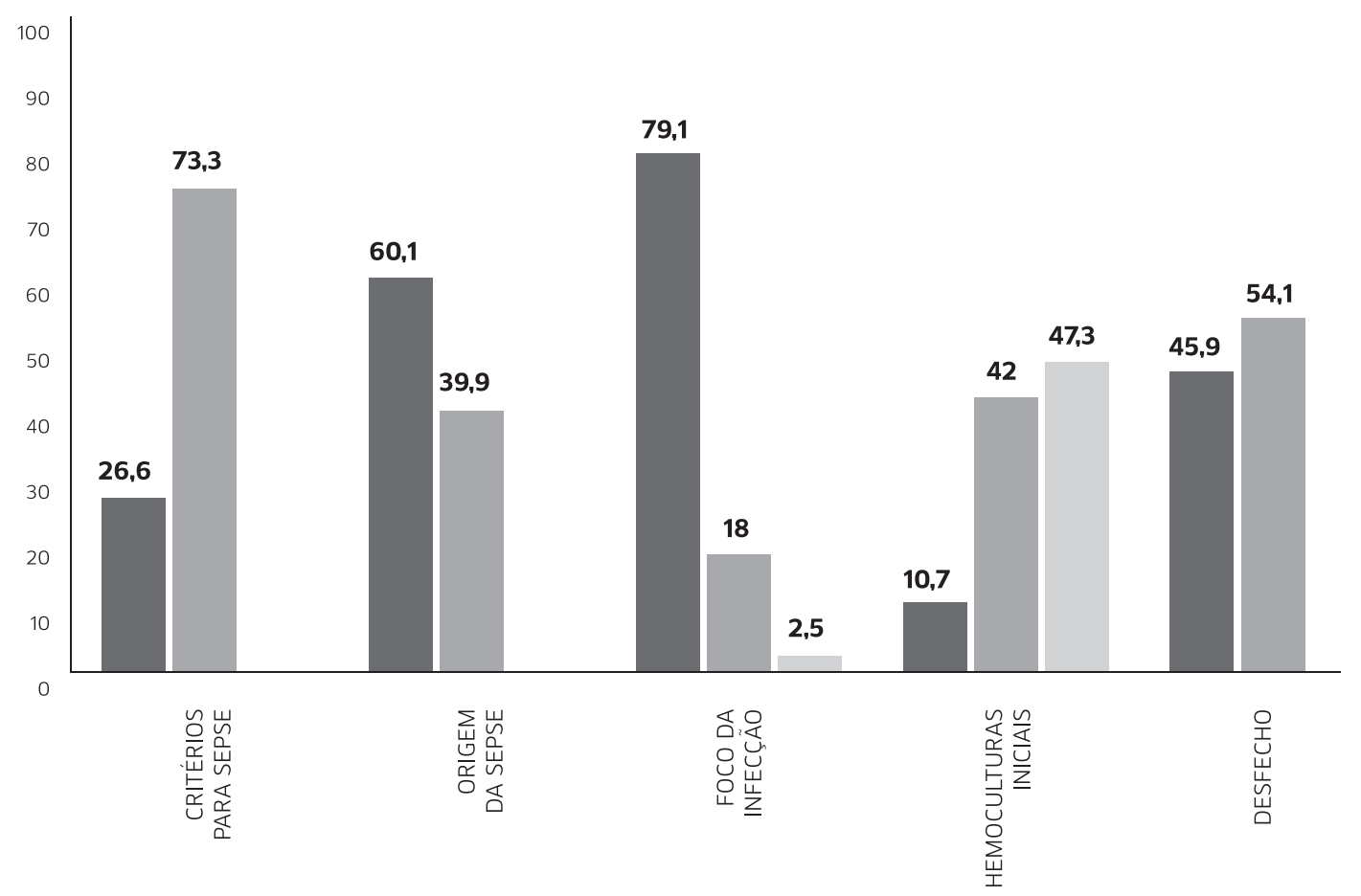

Fonte: Elaborado pelos autores (2018)

Este estudo realizou uma análise dos resultados utilizando o método de tabulação cruzada (Figura 4), comparando o grau de mortalidade e relacionando-os aos seus sintomas e sinais clínicos. Então, verificou-se que a taquicardia e a taquipneia estão associadas ao óbito, pois de um total de 341 pacientes que apresentaram taquicardia, 187 evoluíram a óbito, e dos 260 que apresentaram taquipneia 140 tiveram o mesmo desfecho.
Notou-se também que entre os pacientes que apresentaram leucocitose, 164 foram a óbito. A dosagem da creatinina, marcador importante da disfunção orgânica, mostrou-se elevada, acima de 2.0mg/dl em 262 casos de sepse, e 144 foram a óbito.

Foi possível observar ainda a necessidade de manter suporte de oxigênio para suprir a dessaturação, bem como a administração de 
drogas vasoativas, na intenção de reverter a hipotensão severa causada pelo choque séptico. Entretanto, dos 294 pacientes submetidos ao suporte de oxigênio, 165 foram a óbito. Quanto aos 316 pacientes que receberam droga vasoativa, 173 deles foram a óbito.

Figura 4 Resultados da tabulação cruzada associando sinais e sintomas clínicos com a sepse

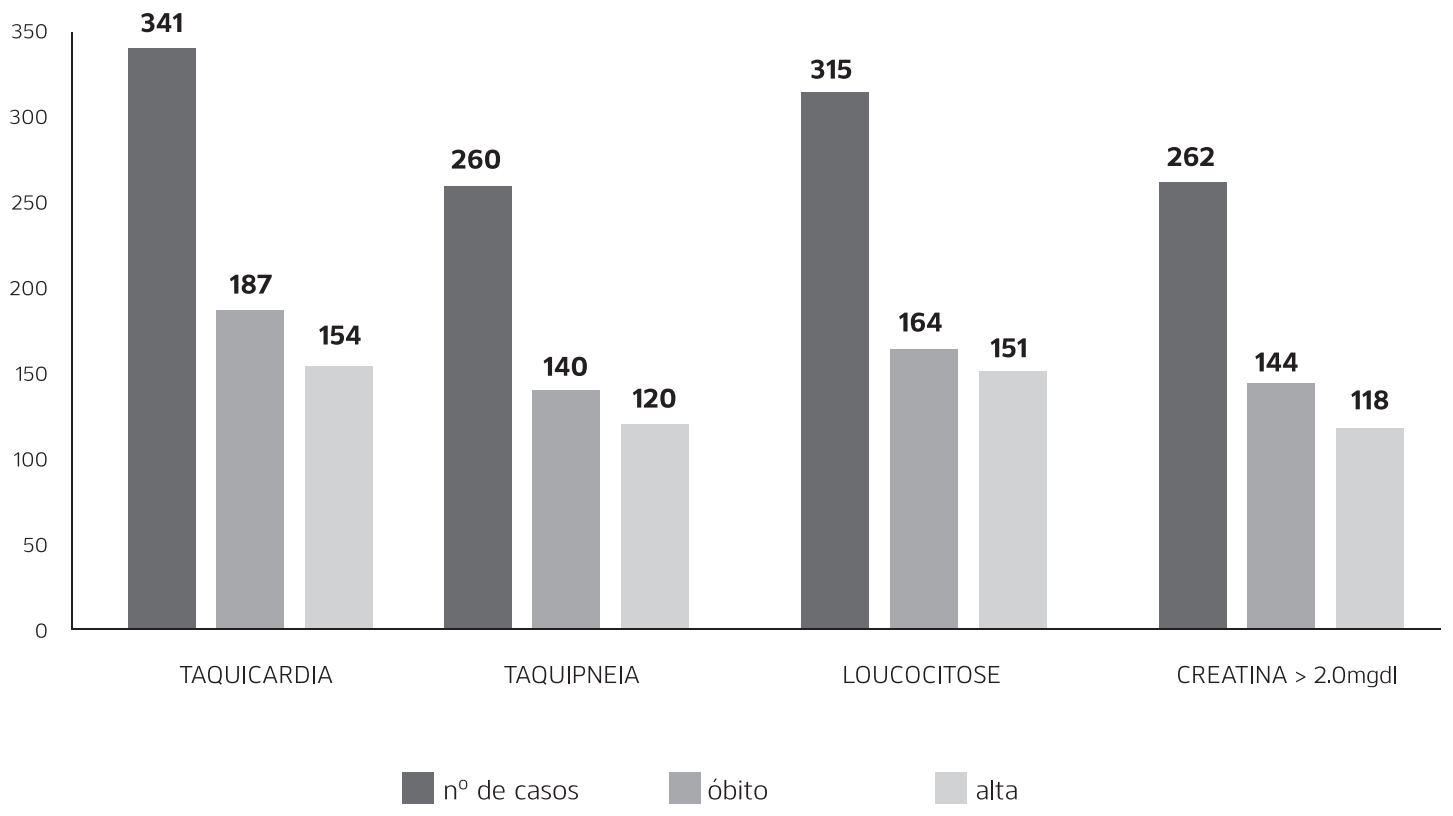

Fonte: Elaborado pelos autores (2018)

É válido enfatizar que tais manifestações clínicas incorrem para a disfunção orgânica no paciente, desta forma, ao analisar todos os casos, foi possivel notar que 399 casos evoluíram com disfunção, e, destes, 218 foram a óbito.

\section{DISCUSSÃO}

No decorrer do desenvolvimento desta pesquisa, bem como na análise dos dados coletados, nota-se o preocupante índice dos casos de sepse, o qual se mostrou elevado, com mortalidade acentuada, afetando principalmente o público masculino com idade superior a 50 anos.

Além disso, outro achado importante foi a quantidade de culturas negativas, a presença de germes multirresistentes, e a não realização desse exame. A cultura de fluidos corporais é essencial para o diagnóstico séptico, pois, além de identificar o agente causador do quadro infeccioso, preconiza o tratamento de forma correta e eficaz ${ }^{8}$.

Estes achados refletem de forma preocupante no âmbito da saúde devido ao fato de que, além do número elevado de óbitos, ocorrem sérias complicações com os pacientes, achado este 
já disposto em outro estudo ${ }^{9}$ que reafirma a gravidade da sepse.

Como descrito no presente estudo, houve a prevalência de indivíduos do sexo masculino e com faixa etária acima dos 50 anos, semelhantemente a um estudo que descreveu a UTI adulto como um setor que admite em maior número pacientes idosos, com idade média de 64,7 anos $^{10}$. Além disso, pesquisas realizadas no Brasil e no mundo evidenciam que a população idosa é predominante nos casos de sepse.

A sepse mostra-se com um número considerável de comorbidades associadas. Nesta pesquisa, menos da metade dos pacientes possuíam doenças crônicas, e dentre os que apresentaram, prevaleceu HAS e diabetes. Estes mesmos dados foram evidenciados em outro estudo ${ }^{2}$, em que a idade média de 62 anos para os pacientes internados em UTIs se refletiu não só no Brasil, mas no mundo todo.

O tipo de internação na UTI mais comum foi de pacientes cirúrgicos e de clínicos. Porém, pesquisa realizada na cidade de Natal (RN) mostrou que somente $20,7 \%$ dos internamentos em uma UTI foram por condições cirúrgicas e 79,6\% foram internadas para tratamento clínico ${ }^{11}$.

Essa diferença, quando se comparam os dois estudos, pode ser explicada pelo fato de que o hospital do presente estudo é referência em cirurgias neurológicas. Os pacientes que passam por cirurgias de alta complexidade necessitam de leitos em UTIs, pois o risco de descompensar a função hemodinâmica é elevado, além dos procedimentos invasivos servirem como forma de exposição a agentes infecciosos ${ }^{12}$.

As etiologias de admissão em UTI são variadas, porém, concomitante com o fato de que o maior número de pacientes é o de idosos, as internações por complicações respiratórias como insuficiência respiratória aguda e broncopneumonia foram as mais frequentes neste estudo. Pesquisas desenvolvidas em hospital filantrópico de São Paulo e em hospital no Ceará, igualmente apontaram que as internações por complicações respiratórias são predominantes em UTIs ${ }^{1,6}$.

Uma grande parte dos pacientes estudada apresentava condições crônicas, como HAS e diabetes, fatores agravantes para o quadro. Estudo feito em 2017 apontou que as comorbidades associadas em mais de $50 \%$ dos pacientes admitidos em uma UTI eram HAS e diabetes ${ }^{12}$. As doenças crônicas servem como fator de predisposição ao agravamento do quadro séptico e estão diretamente associadas ao óbito ${ }^{5}$.

Os sinais e sintomas clínicos da sepse mostraram-se evidentes no estudo em questão, tendo em vista que a maioria dos pacientes apresentou hipertermia, taquicardia e leucocitose. Um estudo feito no ano de 2016 identificou as mesmas alterações em mais de 45\% dos pacientes que desenvolveram sepse', assim como uma pesquisa de 2017, na qual mais de 50\% dos pacientes com quadro séptico apresentaram hipertermia, taquicardia, leucocitose e taquipneia ${ }^{3}$.

As manifestações clínicas da síndrome surgem da sequência deste feedback positivo, ou seja, logo após o início do processo infeccioso primário ocorre a resposta inflamatória subjacente e começam a surgir as disfunções orgânicas que definem o curso da sepse ${ }^{13}$.

As mudanças clínicas que surgem no início do quadro séptico caracterizam-se pela queda da saturação de oxigênio, alteração do nível de consciência - a qual pode ser mensurada a partir da aplicação da escala de coma de Glasgow taquipneia e/ou taquicardia, acidose metabólica devido à elevação do lactato, acidose respiratória, hipertermia e leucocitose ${ }^{14}$.

O choque séptico foi detectado em mais da metade dos pacientes durante a internação na UTI, assim como em uma pesquisa realizada em um hospital de ensino de referência em doenças infecciosas em Belém, Amazônia, em que 57\% dos pacientes com sepse evoluíram com choque séptico ${ }^{5}$.

Apesar dos avanços o diagnóstico precoce 
da sepse continua sendo um grande desafio nas unidades de emergências e de cuidados intensivos, pois sua detecção é de difícil percepção devido à ausência de sintomas e sinais clínicos específicos ${ }^{2}$. As seis primeiras horas após o diagnóstico constituem a janela de oportunidade no tratamento da sepse, e a correta terapia aplicada dentro dessa fase inicial é capaz de reduzir a mortalidade da sepse e o choque séptico em aproximadamente $16 \%{ }^{15}$.

Nesta pesquisa, a origem da infecção teve prevalência comunitária, ou seja, os pacientes já se internaram na UTI apresentando manifestações da sepse. O mesmo foi evidenciado em outra pesquisa realizada em um hospital público, na qual a origem da sepse na maior parte dos casos foi comunitária16.

O foco infeccioso prevalente foi o pulmonar, e o mesmo resultado deu-se em outra pesquisa, na qual o sítio da infecção na maior parte dos casos foi o trato respiratório $(56,2 \%)^{4}$. Levandose em conta que a sepse pode estar relacionada a qualquer foco infeccioso, é preciso lembrar que as infecções mais comumente associadas a sua ocorrência são: a pneumonia, a infecção urinária e a infecção intra-abdominal. A pneumonia, em geral, é o foco responsável por pelo menos metade dos casos de sepse.

A mortalidade elevada é um fator preocupante não só neste estudo, mas no Brasil todo, pois o índice da sepse agrava-se com o passar dos anos. Observa-se também que a taxa de óbito aumenta conforme a faixa etária, ou seja, quanto maior a idade dos pacientes, maior o índice de mortalidade ${ }^{17}$.

Este estudo apresenta algumas limitações, dentre as quais destaca-se o desenho retrospectivo, em que a avaliação clínica dos pacientes ficou restrita às evoluções dos profissionais de saúde do setor, registradas no prontuário. Dessa forma, possiveis fatores como preenchimento incompleto dos prontuários atrelados à falta de notificação dos casos de sepse dificultam estudos com tal metodologia.

Além disso, ao longo do período de pesquisa os critérios diagnósticos de sepse foram alterados ${ }^{18}$. onde parâmetros da síndrome da resposta inflamatória sistêmica (SRIS) não são mais usados para a definição, ou seja, a presença de disfunção orgânica pode representar diagnóstico de sepse, mesmo na ausência de SRIS. A expressão "sepse grave" foi colocada em desuso, ou seja, toda sepse agora é considerada como grave. Dessa forma, no presente estudo as variáveis como a frequência da sepse e suas classificações poderiam sofrer alterações diante da utilização dos critérios recentemente atualizados.

\section{CONSIDERAÇÕES FINAIS}

Constatou-se índice elevado dos casos de sepse. Esse dado é preocupante quando comparado com a literatura atual, pois a taxa de mortalidade mostrou-se elevada, ultrapassando a média geral do país. As complicações da sepse mostraram-se agravantes, com rápida evolução para a disfunção orgânica, tornando os quadros irreversiveis e contribuindo para o óbito.

A presente pesquisa contribui para o âmbito da saúde, pois trouxe dados relevantes frente ao quadro clínico que mais causa óbitos em hospitais, além de tratar da importância da realização de exames como as culturas e do uso correto de antibiótico. Os resultados deste trabalham apontam para a necessidade da realização de novos estudos, tanto retrospectivos quanto prospectivos, para a avaliação integral dos pacientes admitidos em UTI.

Sugere-se uma monitorização e notificação mais eficaz dos casos, combinada com a aplicação de um protocolo unificado, visando à redução da taxa de letalidade por esta síndrome. 


\section{REFERÊNCIAS}

1. Santos AM, Souza GRB, Oliveira AML. Sepse em adultos na unidade de terapia intensiva: caracteristicas clínicas. Arq Med Hosp Fac Cienc Med Santa Casa São Paulo. 2016:61:3-7.

2. Anselmo Júnior E, Dall'Stella DK, Araújo JM, Souza ES, Schuelter-Trevisol F. Incidência de sepse nosocomial em adultos de uma unidade de terapia intensiva, Tubarão SC, em 2013. ACM Arq Catarin Med [Internet]. 2017 [citado em 2019 Out 22];46(4):1726. Disponivel em: http://www.acm.org.br/acm/seer/index.php/ arquivos/article/view/161.

3. Garrido F, Tieppo L, Pereira MDS, Freitas R, Freitas WM, Filipini $R$, et al. Ações do enfermeiro na identificação precoce de alterações sistêmicas causadas pela sepse grave. ABCS Health Sci. 2017:42(1):15-20.

4. Zonta FNS, Velasquez PGA, Velasquez LG, Demetrio LS, Miranda D. Silva MCBD. Características epidemiológicas e clínicas da sepse em um hospital público do Paraná. Rev Epidemiol Controle Infecç. 2018:8(3):224-31.

5. Barros LLS, Maia CSF, Monteiro MC. Fatores de risco associados ao agravamento de sepse em pacientes em Unidade de Terapia Intensiva. Cad Saúde Coletiva. 2016:24(4):388-96.

6. Melo EM, Barbosa AA, Silva JLA, Studart RMB, Lima FET, Veras JEGLF, et al. Clinical outcome of patients on mechanical ventilation in intensive care unit. Rev Enferm UFPE on line. 2015;9(2):610-6.

7. Brasil, Conselho Nacional de Saúde. Resolução $n^{\circ}$ 466, de 12 de dezembro de 2012. Aprova normas regulamentadoras de pesquisas envolvendo seres Humanos [Internet]. Saúde Legis - Sistema de Legislação da Saúde. 2012 [citado em 2019 Out 22]. Disponivel em: https://bvsms.saude.gov.br/bvs/saudelegis/ cns/2013/res0466_12_12_2012.html

8. Carvalho MR, Moreira ICCC, Neta FLA, Guimarães MSO, Viana VGF, Oliveira FW. Incidência de bactérias multirresistentes em uma unidade de terapia intensiva. R Interd. 2015:8(2):75-85.

9 Freitas RB, Santiago MT, Bahia CP. Pereira LP. Mello CMV, Nogueira ACR, et al. Aspectos relevantes da sepse. Rev. Cient. FAGOC Saúde. 2017:1(2): 25-32.

10 Cruz LL, Macedo CC. Perfil epidemiológico da sepse em hospital de referência no interior do Ceará. Id On Line Revista Multidisciplinar e de Psicologia. 2016:10(29):71-99.

11. Bonfada D, Santos MM, Lima KC, Garcia-Altes A. Análise de sobrevida de idosos internados em unidades de terapia intensiva. Rev. Bras. Geriatr. Gerontol. 2017:20(2):198-206.

12. Pauletti M, Otaviano MLPO, Moraes ADST, Schneider DS. Perfil epidemiológico dos pacientes internados em um Centro de Terapia Intensiva. Aletheia. 2017:50(1-2):38-46.

13. Machado FR, Assunção MSC, Cavalcanti AB, Japiassú AM, Azevedo LCP. Oliveira MC. Chegando a um consenso: vantagens e desvantagens do Sepsis 3 considerando paises de recursos limitados. Rev Bras Ter Intensiva. 2016;28(4):361-5.

14. Shankar-Hari M. Phillips GS, Levy ML, Seymour CW, Liu VX, Deutschman CS, et al. Developing a new definition and assessing new clinical criteria for septic shock: for the Third International
Consensus Definitions for Sepsis and Septic Shock (Sepsis-3). JAMA. 2016 Fev 23:315(8):775-87

15. Peninck PP. Machado RC. Aplicação do algoritmo da sepse por enfermeiros na unidade de terapia intensiva. Ver. Rene. 2012:13(1):187-99.

16. Santos AV, Silva AAO, Sousa, ÁFL, Carvalho MM, Carvalho LRB, Moura MEB. Perfil epidemiológico da sepse em um hospital de urgência. Rev. Prev. Infecç. Saúde. 2015:1(1):19-30.

17. Teles LS, Galdino LP, Lima MMP. Santos MM, Cardoso LA. Enfermagem frente à sepse: uma revisão literária. In: Congresso Internacional de Enfermagem; 2017 Maio 9-12; Aracaju-SE. Aracaju: Universidade Tiradentes; 2017. p. 1-4.

18. Singer M, Deutschman CS, Seymour CW, Shankar-Hari M, Annane D. Baver $M$, et al. The Third International Consensus Definitions For Sepsis and Septic Schock (Sepsis-3). JAMA. 2016;315(8):801-10 